\title{
Adaptive Transition of Aquaporin 5 Expression and Localization during Preimplantation Embryo Development by In Vitro Culture
}

\author{
Jae-Won Park ${ }^{-1}$, Yun Kyung $\operatorname{Shin}^{-2}$ and ${ }^{\dagger}$ Yong-Pil Choen ${ }^{1}$ \\ ${ }^{I}$ Division of Developmental Biology and Physiology, School of Biosciences and Chemistry, Institute for Basic Sciences, \\ Sungshin University, Seoul 147-742, Korea \\ ${ }^{2}$ Aquaculture Management Division, Aquaculture Research Institute, NFRDI, Busan 619-902, Korea
}

\begin{abstract}
Adaptive development of early stage embryo is well established and recently it is explored that the mammalian embryos also have adaptive ability to the stressful environment. However, the mechanisms are largely unknown. In this study, to evaluate the possible role of aquaporin in early embryo developmental adaptation, the expression of aquaporin (AQP) 5 gene which is detected during early development were examined by the environmental condition. To compare expression patterns between in vivo and in vitro, we conducted quantitative RT-PCR and analyzed localization of the AQP5 by whole mount immunofluorescence. At in vivo condition, Aqp5 expressed in oocyte and in all the stages of preimplantation embryo. It showed peak at 2-cell stage and decreased continuously until morula stage. At in vitro condition, Aqp5 expression pattern was similar with in vivo embryos. It expressed both at embryonic genome activation phase and second midpreimplantation gene activation phase, but the fold changes were modified between in vivo embryos and in vitro embryos. During in vivo development, AQP5 was mainly localized in apical membrane of blastomeres of 4-cell and 8-cell stage embryos, and then it was localized in cytoplasm. However, the main localization area of AQP5 was dramatically shifted after 8-cell stage from cytoplasm to nucleus by in vitro development. Those results explore the modification of Aqp5 expression levels and location of its final products by in vitro culture. It suggests that expression of Aqp5 and the roles of AQP5 in homeostasis can be modulated by in vitro culture, and that early stage embryos can develop successfully by themselves adapting to their condition through modulation of the specific gene expression and localization.
\end{abstract}

Key words : Aquaporin 5, Preimplantation embryo, Localization, Adapation, In vitro cultrue

\section{INTRODUCTION}

Developing of media and culture methods make a way which support successful mammalian embryo development to the blastocoel stage within a given time, although the environment is different from their natural courses. Some of reports, which were concerned with the adaptational development of early stage embryo, evaluated the phe- nomena in various animals. However, it is not well determined in mammalian embryos. Recently, adaptation of early stage embryo development to the stressful hyper-osmotic stress were studied by Bell et al. (2009) and revealed an expression modification of some genes.

Homeostasis in cytoplasm is maintained through exchanges with external environment. Small changes in cell volume and pressure can serve as important signals for

\footnotetext{
Manuscript received 21 July 2014, Received in revised form 30 July 2014, Accepted 8 August 2014

† Corresponding Author : Yong-Pil Cheon, Division of Developmental Biology and Physiology, School of Biosciences and Chemistry, Sungshin University, Seoul 147-742, Korea. Tel. : +82-2-920-7639, 2766, Fax : +82-2-920-2047, E-mail : ypcheon@sungshin.ac.kr JWP and YKS contributed equally to this work.

This is an Open Access article distributed under the terms of the Creative Commons Attribution Non-Commercial License (http:// creativecommons.org/licenses/by-nc/3.0) which permits unrestricted non-commercial use, distribution, and reproduction in any medium, provided the original work is properly cited.
} 
cellular responses such as proliferation, death, and migration. Therefore, cell must adapt to osmotic shocks and actively adjust the adaptation levels (Jiang \& Sun, 2013). Homeostatic internal environment in hydrostatic and osmotic pressure of egg and preimplantation embryo is partly depending in the amount of water, ion, and nutrients (Cheon, 2008; Yun et al., 2013). Therefore, mechanosensitive channels, active ion pumps, and water channels are a key to understanding the overall mechanical response for cellularization during early stage embryo development.

AQPs are transmembrane channel proteins within cell, that function as water channels allowing water to flow rapidly across the membrane in the direction of osmotic gradients (Shiels \& Griffin, 1993). In mammals, thirteen AQPs (AQP0-12) are revealed and distributed in different organs (Hua et al., 2013). AQPs can be distinguished by their sequence homology and permeable functions as following (Gonen \& Walz, 2006): AQP0, -1, -2, -4, -5, -6, and -8 are aquaporins and highly selective for water molecules, although AQP6 and AQP8 only belong to this group based on their sequence, since AQP6 is an anion channel (Yasui et al., 1999) and AQP8 is permeable to urea (Ishibashi et al., 1997). AQP 3, 7, 9, and 10 are aquaglyceroporins allowing passage of small solute (for example, glycerol, urea, purines, pyrimidines, carbamides, and polyols) with AQP9 exhibiting the broadest substrate specificity (Borgnia et al, 1999; Gonen \& Walz, 2006; Ishibashi et al, 2002; Itoh et al., 2005). In the mouse, AQP 10 gene is a pseudogene (Morinaga et al., 2002). The substrate specificities for the superaquaporins AQP11 and AQP12 have not yet fully determined (Ishibashi et al, 2002, 2014).

AQP5 can be detected in uterus, cervix, oviduct, embryo, and epidydymis (Huang et al., 2006). AQP5 is highly permeable to water but not to small organic and inorganic molecules. It plays a role in regulating membrane water permeability and in maintaining proper osmolarity of the secreted saliva and lens (Kumari \& Varadaraj, 2013; Ma et al., 1999). In embryo development, it has been suggested that AQPs are important in early stage embryo development, especially in blastocoel formation (Offenberg et al., 2000) although AQP5 KO mice are prenatal survival (Krane et al., 2001). Besides, the expression profiling and mechanisms AQP5 are largely unknown during development of preimplantation embryos. Previously, our laboratory screened the expression of the AQPs family during early stage embryos and Aqp5 transcripts were detected. In this study, to know the possible role of Apq5 in the development of preimplantation embryo, we evaluate the expression and location modification of Aqp5 transcripts and proteins by in vitro culture.

\section{MATERIALS AND METHODS}

\section{Experimental animals}

All experiment animals were maintained and treated according to the Guide for the Care and Use of Laboratory animals published by National Institutes of Health and under the Experimental Animals Committee of Sungshin University. Animals were maintained under standard conditions at animal house in Sungshin university with diurnal rhythm kept under the $14 \mathrm{~L}: 10 \mathrm{D}$ schedule with light-on at 06:00 and clean room system. Animals were fed a standard rodent diet and water ad libitum from weaning at 21 days after birth.

\section{Superovulation induction and embryo collection}

Six to eight week-old female CD-1 mice were used in this study. Animals were injected with 5 IU/0.1mL PMSG (Sigma) followed by $5 \mathrm{IU} / 0.1 \mathrm{~mL}$ hCG (Sigma) $48 \mathrm{hr}$ later, and mated with CD-1 males. Next morning, the day of vaginal plug was designated Day 1 of pregnancy. Time post-hCG was standard to measure the developmental stage embryo which were collected at $18 \mathrm{hr}$ unfertilized egg (UF); 21-24 hr pronucleus stage embryo (PN); $48 \mathrm{hr}$, 
2-cell (2-C); 60 hr, 4-cell (4-C); 65-68 hr 8-cell (8-C); 80$85 \mathrm{hr}$, morula (Mor); 96-100 hr, blastocyst (Bla). To collect in vivo embryos, we flushed the oviduct and uterus using flushing medium $0.4 \%$ BSA BWW. Flushed 2-cell stage embryo were cultured to blastocyst. Collected embryos were quick-frozen in a small amount of medium using liquid nitrogen. They were stored at $-80^{\circ} \mathrm{C}$ until RNA extraction.

\section{Embryo culture}

The collected healthy two cell embryos were cultured in the $10 \mu \mathrm{l}$ microdrops of BWW medium contacting $0.4 \%$ bovine serum albumin (BSA). The embryo were incubated at $37^{\circ} \mathrm{C}$ with $5 \% \mathrm{CO}_{2}$ in air until $120 \mathrm{hr}$ post hCG injection. The embryo development was evaluated every 12 or $24 \mathrm{hr}$ under the inverted microscope (Olympus, $1 \times 70$ ).

\section{Total RNA extraction and first strand cDNA} synthesis

Total RNA of embryos was extracted by using RNeasy $\mathbb{}$ Micro Kit (QIAGEN, CA USA) according to the manual of manufacturer. Total RNA $5 \mu \mathrm{g}$ were used to perform reverse transcription. First strand cDNA synthesis was conducted by AffinityScript cDNA Synthesis Kit (Agilent, CA, USA) according to the manufacturer's instructions. Briefly, reaction reagents are total RNA $5 \mu \mathrm{g}, 5.0 \mu \mathrm{L}$ Accuscript buffer $(10 \times), 6.0 \mu \mathrm{L}$ oligo dT primer $(0.5 \mu \mathrm{g} / \mu \mathrm{L}), 1.0 \mu \mathrm{L}$ random primer $(0.1 \mu \mathrm{g} / \mu \mathrm{L}), 2 \mu \mathrm{L}$ dNTP mix $(100 \mathrm{mM}), 1$ $\mu \mathrm{L}$ RNase-free water. Reaction mixture was incubated at $65^{\circ} \mathrm{C}$ for $5 \mathrm{~min}$, placed the tube at RT to allow the primers to anneal to RNA for 10 min and then, added $4.0 \mu \mathrm{L}$ DTT (100 mM), $1 \mu \mathrm{L}$ Accuscript multiple temperature RT, $2 \mu \mathrm{L}$ RNase block ribonuclease inhibitor $(40 \mathrm{U} / \mathrm{mL})$. The mixture was incubated at $42^{\circ} \mathrm{C}$ for $1 \mathrm{hr}$ to synthesis and then $72^{\circ} \mathrm{C}$ for 15 min to terminate cDNA synthesis.

\section{Real Time RT-PCR}

Transcripts of target gene were amplified using real time PCR (Takara, TP800) and the specific primer (Table 1). For quantitative RT-PCR (qPCR) was performed using SYBR Premix Ex Taq ${ }^{\mathrm{TM}}$ (TakaRa, Japan) and Thermal Cycler Dice Real Time System TP800 (TaKaRa, Japan) using ribosomal protein, 36B4 as internal control. Each reaction was run in triplicate and consisted of $1.0 \mu \mathrm{L}$ cDNA. Dissociation curves were run on all reactions to ensure amplification of a single product with the appropriate melting temperature. The thermal cycling conditions were : $95^{\circ} \mathrm{C}$ for $1 \mathrm{~min}, 59^{\circ} \mathrm{C}$ for $30 \mathrm{~s}, 72^{\circ} \mathrm{C}$ for $1 \mathrm{~min}$, and for 45 cycles Data were analyzed by the $\Delta \Delta$ comparative threshold cycle (CT) method.

\section{Whole mount immunofluorochemistry of the mouse} embryo

The in vivo and in vitro cultured embryos were fixed in 4\% paraformaldehyde in PBS containing 3.7\% picric acid in phosphate-buffered saline (PBS) for $30 \mathrm{~min}$ and then, washed PBS. Permeablization was conducted by $0.2 \%$ PBST (PBS containing $0.2 \%$ Triton X-100) for $3 \mathrm{hr}$. The embryos were blocked in $0.1 \%$ PBST containing $10 \%$ normal serum for $1 \mathrm{hr}$ at RT. After then, the embryos were

Table 1. Sequences of sense (S) and antisense (AS) primers (5'-3') for real-time RT-PCR

\begin{tabular}{ccl}
\hline \hline Genes & Gene bank access No. & \multicolumn{1}{c}{ Sequneces } \\
\hline \multirow{2}{*}{ Aqp5 } & NM_009701.4 & $\begin{array}{l}\text { S : GAA GAA GGA GGT GTG TTC AGT TGC } \\
\text { AS : TGG TGT TGT GTT GCT GAG C }\end{array}$ \\
\hline \multirow{2}{*}{ Ppia } & NM_008907.1 & S : CCACCGTGTTCTTCGACATCA \\
& AS : GATGCCAGGACCTGTATGCTTTAG \\
\hline
\end{tabular}


incubated with rabbit polyclonal anti-AQP5 (Santa Cruz Biotechnology, Santa Cruz, CA, USA) at a 1:200 dilution at $4^{\circ} \mathrm{C}$ overnight. Washed embryos were incubated with fluorescence 2nd antibody Cy ${ }^{\mathrm{TM}} 3$ conjugated AffiniPure (Jackson ImmunoResearch, USA) diluted 1:200 in PBS containing 2\% BSA for $2 \mathrm{hr}$ at RT. The nuclear of embryos was staining with Hochest33238 $10 \mathrm{~min}$. Dot slide were used for mounting. For negative controls, we deleted primary antibodies. Finally, fluorescent images were analyzed by Zeiss LSM 700 laser scanning microscopes with ZEN software.

\section{Statistics}

Quantitative RT-PCR was conducted at least in triplicate. Differences in the mean values of AQP cDNA copies in the in vivo and in vitro embryos were analyzed using an unpaired $t$-test and ANOVA. Results were presented as Mean \pm SD. Immunofluorescence staining was performed to each group that included in 5 ea embryos.

\section{RESULTS}

\section{Expression patterns of Aqp5 transcript in vivo}

We investigated the change of Aqp mRNA levels in in vivo embryo by measuring the quantitative RT-PCR. During development in vivo condition, Aqp5 mRNA was detected in oocyte, pronucleus stage embryo and all preimplatation stage embryos (Fig. 1). Its expression peaked at 2-cell stage. At morula stage, its expression was dramatically lower than the other stages. Its expression levels were recovered at blastocyst stage (Fig. 1). These results showed that embryonic Aqp5 expression increased at 2-cell and blastocyst stages. It means that there are 2 time points in expression of zygotic Aqp5 during early stage development.

2. Differences in the expression patterns and levels of Aqp5 transcripts in vitro

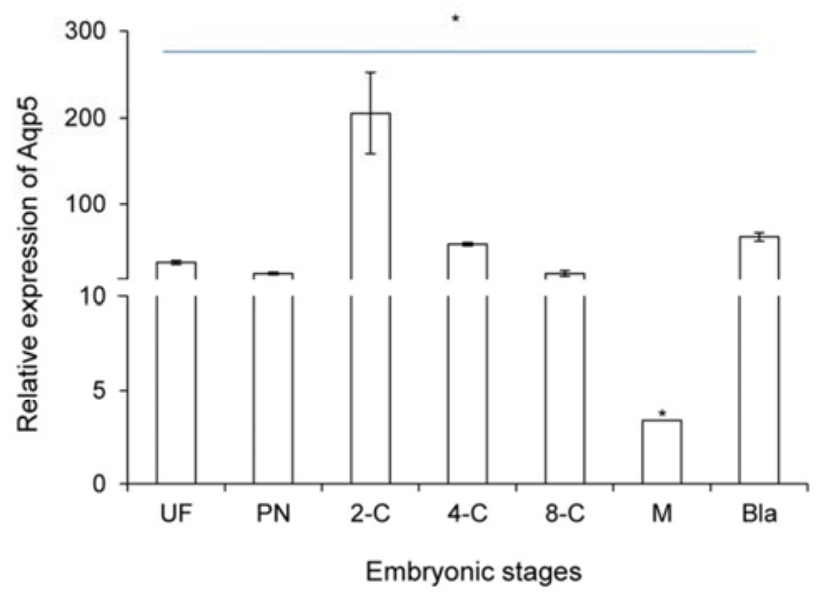

Fig. 1. Relative Aqp5 mRNA levels in preimplantation stage embryos in vivo. Real-time RT-PCR was used to determine the relative mRNA levels of Aqp5 in unfertilized oocyte (UF), pronucleus stage unfertilized egg (PN), 2-cell (2-C), 4-cell (4-C), 8cell (8-C), morula (Mor), and blastocyst (Bla) which were developed in vivo. Ten embryos were used for real-time RT-PCR per embryonic stage and repeated 3 times in all stages. * Significant difference among stages $(p<0.05$, ANOVA).

Under the $\mathrm{BWW}, 37^{\circ} \mathrm{C}, 5 \% \mathrm{CO}_{2}$ in $>95 \%$ humidified air culture condition, the Aqp5 transcript was detected in all the stages examined. Aqp5 mRNA expression patterns were similar to in vivo, but relative fold change was higher than in vivo (Fig. 2). It means that the expression patterns were not changed both in Aqp5 expression but the levels of expression or degradation were modified by in vitro culture.

3. Localization of AQP5 in in vivo early stage embryos and modification by in vitro culture

To evaluate the possible role of AQP5 in early embryo, we localized these proteins with whole mount immunefluorescence method. Whole mount immunefluorescence analysis confirmed expression of AQP5 in vivo and in vitro mouse preimplantation embryo. AQP5 was mainly localized in apical membrane of blastomeres and the levels 


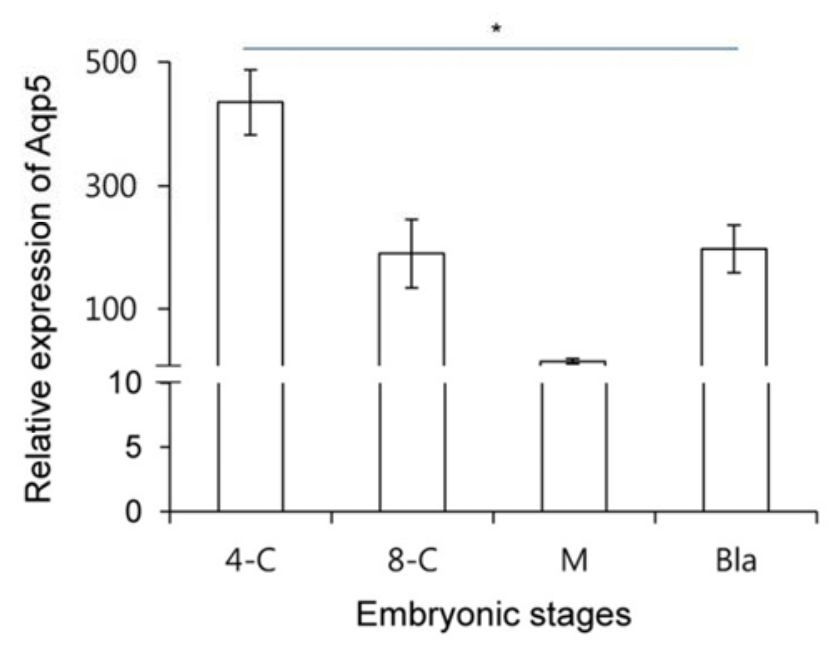

Fig. 2. Relative Aqp5 mRNA levels in preimplantation stage embryos which were cultured in vitro. Realtime RT-PCR was used to determine the relative mRNA levels of Aqp5 in 4-cell (4-C), 8-cell (8-C), morula (Mor), and blastocyst (Bla) which were cultured in vitro from 2-cell stage. Ten embryos were used for real-time RT-PCR per embryonic stage and repeated 3 times in all stages. * Significant difference among stages ( $p<0.05$, ANOVA).

were similar with that of Aqp5 mRNA. During blastocyst, it was localized in cytoplasm of trophectoderm (Fig. 3A-I). During in vitro culture, AQP5 protein localization was mainly localized in apical membrane at 4-cell and 8-cell stages likes in in vivo embryos, but its localization shifted from cytoplasm to nucleus (Fig. 3A'-I'). It was totally different from the same stage in vivo embryo.

\section{DISCUSSION}

This study firstly validated the expression of Aqp5 and localization of their products in the all preimplanation stage embryos. The AQP5 was detected in all preimplantation stages. Previously mRNAs encoding AQP1, $-3,-5,-6,-7$, and -9 were analyzed by Offenberg et al. (2000) in preimplantation mouse embryo. Here, we clearly evaluated their expression in all early stages. Aqp5 gene transcripts were detected in oocytes. Aqp5 was sharply expressed from 2-

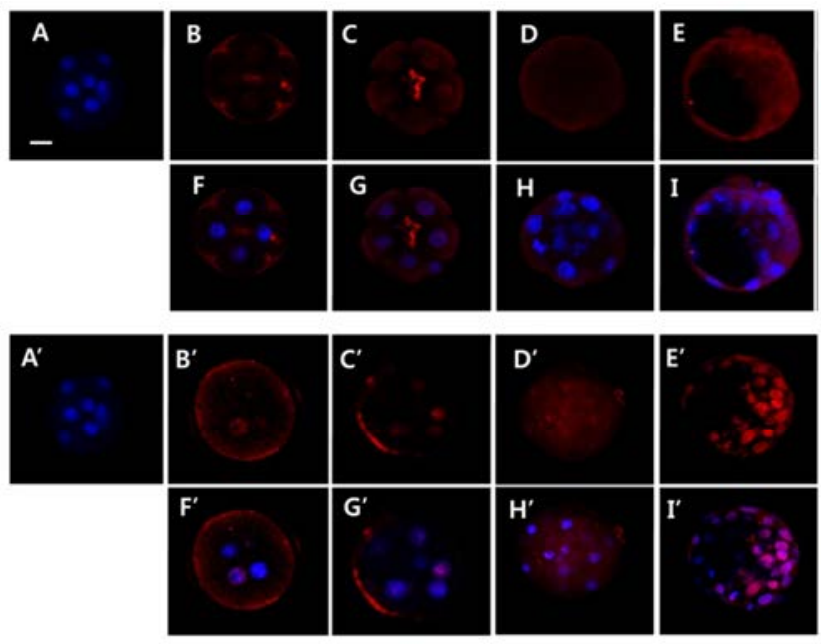

Fig. 3. Laser scanning confocal microscopy image of AQP5 in vivo (A-I) and in vitro (A'-I') developed embryos. The localization of AQP5 in embryo was examined by whole mount immunofluorescence. A and A': negative control; $\mathrm{B}$ and $\mathrm{B}$ ': 4-cell stage embryo; $\mathrm{C}$ and C': 8-cell stage embryo; D and D': morula stage emryo; E and E': blastocyst stage embryo; A$\mathrm{D}$ and A'-D': red (anti-AQP5); F-I and F'-I': merged image (blue: Hochest33238); A-I: in vivo embryos; A'-I': in vitro cultured embryos from 2cell stage; scale bar $=20 \mu \mathrm{m}$.

cell stage and decreased until morula stage. It expressed sharply again at blastocyst stage. These results explored that maternal Aqp5 transcript exist until 2-cell stage and zygotic Aqp5 expression occurs two times during preimplantation stage. First is at embryonic genome activation phase and second is at second mid-preimplantation gene activation phase (Bell et al., 2008). It suggested that the role of Aqp5 product will be different between the expression phases.

On the other hand, interestingly, their expression profiles were modified by in vitro culture. Aqp5 transcript was dramatically increased by in vitro culture. But sharp increase of the expression of zygotic Aqp5 transcripts was maintained at blastocyst stage both in vivo and in vitro. So far, the suggested role of AQPs in early stage embryos is the water transport for developmental transition of preim- 
plantation embryo. Developmental transition to blastocyst stage is defined by the appearance of a fluid-filled cavity (Watson \& Barcroft, 2001). Critical gene families controlling these events include: the E-cadherin-catenin cell adhesion family, the tight junction gene family, the Na/K-ATPase gene family and perhaps the Aqp gene family (Andrew \& Barcroft, 2001). Based on it, it is possible that reappearance of zygotic Aqp5 expression and their modulation at second mid-preimplantation gene activation phase by in vitro culture is for the supporting the blastocoel formation, although more studies are needed.

In addition, the expression patterns of Aqp5 during preimplantation stages were not changed by in vitro culture. The zygotic Aqp5 transcript showed high levels at maternal-zygotic transition period and keeping the patterns of their transcription were observed in in vitro cultured embryos. AQPs are one of the key molecules for cellular osmolality regulation because AQPs transport water, glycerol, $\mathrm{CO}_{2}$, ammonia, urea, and hydrogen peroxide across the membrane (Carbrey \& Agre, 2009). Besides, AQPs are suggested as key molecules for energy and metabolic homeostasis. Cellular volume regulation by osmosis is a key factor for survival and other cellular responses in eukaryotic cells (Jiang \& Sun, 2013). Physicochemical environmental changes are a cause of physiological adaptation of cells and tissues.

Glycerol constitutes important metabolites as a substrate for de novo synthesis of triacylglycerols and glucose as well as an energy substrate to produce ATP. In this sense the control of glycerol influx/efflux in metabolic organs by aquaglyceroporins plays crucial role (Calamita et al., 2012; Méndez-Giménez et al., 2014). Because Aqp5 products are not included in aquaglyceroporins, its role in development of preimplantation stage embryo may be osmolality regulation. The known main function of AQP5 is regulation of membrane water permeability and maintaining proper osmolarity (Kumari \& Varadaraj, 2013; Ma et al., 1999). Based on them, it is suggested that Aqp5 may be an osmotic adaption to in vitro culture condition.

AQP5 role during preimplantation stage embryos were assumed with its localization. AQP5 localized mainly in apical membrane at 4-cell and 8-cell stages, after then it localization shifted from membrane to cytoplasm. Its location was not changed by in vitro culture. However, interestingly, it is mainly localized in the nucleus of blastocyst by in vitro culture. Previously, it is known that phosphorylation of AQP5 results in internalization of the protein from plasma membrane (Kumari et al., 2012), but it is not known how the AQP5 translocate into the nucleus. It suggested that AQP5 may be involved in water transprot at apical membrane of blastomeres at 4-cell and 8-cell but after then it may work in cytoplasm. In addition, it means that the role of AQP5 at blastocyst stage may differ between in vivo and in vitro.

In summary, the expression of Aqp5 gene expression during early stage embryos showed dramatic adaptation to the changed environment. Location of AQP5 is also modified by in vitro culture. It is known that AQP5 is aquaporin and highly selective for water molecules. Put together, it suggests that AQP5 may be involved in the homeostasis of blastomere through water transport regulation at apical membrane of blastomeres at 4-cell and 8-cell, but not at morula and blastocyst. It also suggested that the expression of Aqp5 and its roles can be modulated by environmental changes, and early stage embryos can develop successfully by themselves adapting to their condition through modulation of the specific gene expression and localization. Furthermore, these finding can be applied to getting an implantation competent blastocyst. This study emphasizes the need to further appreciate and consider the adaptation phenomena in early stage mammalian embryo.

\section{ACKNOWLEDGEMENT}


This works was supported by Basic Science Research Program through the National Research Foundation of Korea (NRF) funded by the Ministry of Education, Science and Technology (NRF-2012R1A1A2003084) and National Fisheries Research and Development Institute (NFRDI) (AQ-2014-90).

\section{REFERENGES}

Andrew AJ, Barcroft LC (2001) Regulation of blastocyst formation. Front Biosci 1:D708-730.

Bell CE, Calder MD, Watson AJ (2008) Genomic RNA profiling and the programme controlling preimplantation mammalian development. Mol Hum Reprod 14:691701.

Bell CE, Lariviere NMK, Watson PH, Watson AJ (2009) Mitogen-activated protein kinase (MAPK) pathways mediate embryonic responses to culture medium osmolarity by regulating aquaporin 3 and 9 expression and localization, as well as embyonic apoptosis. Hum Reprod 24:1373-1386.

Borgnia M, Nielsen S, Engel A, Agre P (1999) Cellular and molecular biology of the aquaporin water channels. Annu Rev Biochem 68:425-458.

Calamita G, Gena P, Ferri D, Rosito A, Rojek A, Nielsen S, marinelli RA, Frühbeck G, Gvelto M (2012) Biophysical assessment of aquaporin-9 as principal facilitative pathway in mouse liver imort of glucogenetic glycerol. Biol Cell 104:342-351.

Carbrey JM, Agre P (2009) Discovery of the aquaporins and development of the field. Handb Exp Pharmacol 190:3-28.

Cheon YP (2008) Carbohydrate metabolism in preimplantation stage embryos and the role of metabolites. Dev Reprod $12: 19-30$

Gonen T, Waltz T (2006) The structure of aquaporins. Q Rev Biophys 39:361-396.
Hua Y, Jiang W, Zhang W, Shen Q, Chen M, Zhu X (2013) Expression and significance of aquaporins during pregnancy. Front Biosci 18:1373-1383.

Huang HF, He RH, Sun CC, Zhang Y, Meng QX, Ma YY (2006) Funciton of aquaporins in female and male reproductive systems. Hum Reprod Updata 12:785-795.

Ishibashi K, Kuwahara M, Gu Y, Kageyama Y, Tohsaka A, Suzuki F, Marumo F, Sasaki S (1997) Cloning and funcitonal expression of a new water channel abundantly expressed in the testis permeable to water, glycerol, and urea. J Biol Chem 272:20782-20789.

Ishibashi K, Morinaga T, Kuwahara M, Sasaki S, Imai M (2002) Cloning and identificaiton of a new member of water channel (AQP10) as an aquaglyceroporin. Biochem Biophys Acta 1576:335-340.

Itoh T, Rai T, Kuwahara M, Ko SB, Uchida S, Sasaki S, Ishibashi K (2005) Identification of a novel aquaporin, AQP12, expressed in pancreatic acinar cells. Biochem Biophys Res Commun 330:832-838.

Jiang H, Sun SX (2013) Cellular pressure and volume regulation and implications for cell mechanics. Biophys $\mathrm{J}$ 105:609-619.

Krane CM, Melvin JE, Nguyen HV, Richardson L, Towne JE, Doetschman T, Menon AG (2001) Salivary acinar cells from aquaporin 5-deficient mice have decreased membrane water permeaility and altered cell volume regulaiton. J Biol Chem 276:23413-23420.

Kumari SS, Varadaraj K (2013) Aquaporin 5 knockout mouse lens develops hyperglycemic cataract. Biochem Biophys Res Com 441:333-338.

Kumari SS, Varadaraj M, Yerramilli VS, Menon AG, Varadaraj K (2012) Spatial expression of aquaporin 5 in mammalian cornea and lens and regulation of its localization by phosphokinase A. Mol Vis 18:957-967.

Ma T, Song Y, Gillespie A, Carlson EJ, Epstein CJ, Verkman AS (1999) Defective secretion of saliva in transgenic mice lacking aquaporin-5 water channels. J Biol Chem 
274:20071-20074.

Méndez-Giménez L, Rodriguez A, Balaguer I, Frühbeck G (2014) Role of aqualyeroporins and caveolins in energy and metabolic homeostasis. Mol Cell Endocrinol S03037207(14)00200-7. Doi: 10.1016./j.mce.201406.017.

Morinaga T, Nakakoshi M, Hirao A, Imai M, Ishibashi K (2002). Mouse aquaporin 10 gene (AQP10) is a pseudogene. Biochem Biophys Res Commun 294:630-634.

Offenberg H, Barcroft LC, Caveney A, Viuff D, Thomsen PD, Watson AJ (2000) mRNAs encoding aquaporins anre present during murine preimplantation development. Mol Reprod Dev 57:323-330.

Shiels A, Griffin CS (1993) Aberrant expression of the gene for lens major intrinsic protein in the CAT mouse. Curr Eye Res 12:913-921.

Watson AJ, Barcroft LC (2001) Regulation of blastocyst formation. Front Biosci 6:D708-D730.

Yasui M, Hazama A, Kwon TH, Nielsen S, Guggino WB, Agre P (1999) Rapid gating and anion permeability of an intracellular aquaporin. Nature 402:184-187.

Yun X, Tan YJ, Xiong YM, Huang YT, Hu XL, Lu YC, Ye YH, Wang TT, Zhang D, Jin F, Huang HF, Sheng JZ (2013) Expression of aquaporins in human embryos and potential role of AQP3 and AQP7 in preimplantation mouse embryo development. Cell Physiol Biochem 31: 649- 658 . 